\title{
Mc Gregor Flap for Lower Eyelid Defect
}

\author{
Dr.Rishiraj Yadav,Dr. M.F. Shaikh,Dr.Salesh Kumar Soni,Dr.Hardik Dodia \\ ( $3^{\text {rd }}$ year resident, Department of burns and plastic surgery, B. J. Medical College/ Gujarat University, India) \\ (Head of the department, Department of burns and plastic surgery, B. J. Medical College/ Gujarat University, \\ India) \\ ( $3^{\text {rd }}$ year resident, Department of burns and plastic surgery, B. J. Medical College/ Gujarat University, India) \\ ( $2^{\text {nd }}$ year resident, Department of burns and plastic surgery, B. J. Medical College/ Gujarat University, India)
}

\begin{abstract}
:
Introduction: The reconstruction of a full thickness loss of the lower eyelid after neoplasm excision is an immediate functional requisite, since the eyelids are organs of primary importance for the protection of the eyeball. There are various techniques of closing the defect. Here I am describing Mc gregor flap technique for coverage of lower lid defect.
\end{abstract}

Keywords: Lower eyelid defect, BCC of lateral canthus of eyelid, Mc Gregor Flap, Lower eyelid defect coverage, Cheek advancement flap with Z plasty.

\section{Introduction}

The reconstruction of a full thickness loss of the lower eyelid after neoplasm excision is an immediate functional requisite, since the eyelids are organs of primary importance for the protection of the eyeball. There are various techniques of closing the defect. Here I am describing Mc gregor flap technique for closure of lower lid defect. This flap consists of an inferiorly based transposed flap raised on the skin area below and lateral to the outer canthus, and it is moved medially to close V-shaped full-thickness defects of the lower eyelid. Transfer of the flap leaves a triangular secondary defect laterally, and this is closed using a Z-plasty.

\section{Flap Design And Dimensions}

Before raising the flap, the lines along which the various incisions will be made are drawn out on the skin. With the V-shaped defect outlined on the eyelid, the line that indicates the upper border of the transposed flap extends laterally from the outer canthus, continuing the upward curve of the margin of the lower eyelid, to just short of the temple hairline. The upward curve is required to provide an adequate vertical height of the reconstructed eyelid segment and to avoid ectropion.

From the lateral extremity of this line, a further line is drawn in a generally downward direction parallel to the lateral limb of the $\mathrm{V}$ on the eyelid, the angle between the two lines being approximately $60^{\circ}$. The effect is to outline a flap with the overall shape of a parallelogram. As already indicated, transfer of the flap medially leaves a triangular defect that is closed by a Z-plasty. This is designed at this stage, before any skin incisions are made, constructed around the lateral part of the line representing the upper margin of the flap. This acts as the common limb of the $\mathrm{Z}$, it and the line representing the lateral margin of the flap together forming one of the Z-plasty flaps. Design of the Z-plasty is completed with the drawing of a line that runs upward and laterally from the upper margin of the flap, outlining the second Z-plasty flap.

\section{Indications}

The reconstruction that uses this flap is primarily applicable to full-thickness defects of the lower eyelid that can be created in the form of a V, although, as discussed later, the principle also can be applied to comparable defects of the upper eyelid. This flap can be used for defects of up to two-thirds of the breadth of the lower lid, canthus to canthus, but defects greater in breadth are unsuitable. The technique is one of the triad of reconstructions used to treat full-thickness $\mathrm{V}$-shaped defects of increasing breadth. With the narrow $\mathrm{V}$, the defect can be closed directly; where the defect is broader and direct closure would be under tension, reduction of tension can be provided by dividing the slip of the lateral canthal ligament to the lower eyelid-lateral canthotomy. It is when the defect is still too broad for lateral canthotomy alone that the transposed flap has to be advanced medially to bring together the two limbs of the $\mathrm{V}$ and close the defect.

The method is easiest to carry out technically when an element of tarsal plate is present on both sides of the defect. The presence of the plate provides a good base for suturing the two margins of the $\mathrm{V}$ together, and while the method can be used for defects that abut on one or the other canthus, the absence of tarsal plate on one side of the defect makes secure closure of the defect more difficult. 


\section{Operative Technique}

With the V excision of the full thickness of the eyelid completed, the flap, as outlined, is raised at the face lift level lateral to the orbicularis oculi. When the orbicularis is reached, the plane of elevation becomes deep to the muscle, the muscle being advanced as part of the flap. In the process of elevating the flap, the slip of the lateral canthal ligament to the lower eyelid is divided. This division can be carried out as a formal step, but the ligament can just as effectively be felt as a resistance to medial flap advancement and be divided. With a skin hook to gently pull the flap medially, sites of resistance to advancement can be identified with the finger and divided with scissors. In the process, much of the lower border of orbicularis is divided, as well as the orbital septum, neither structure being divided as a formal, visualized step, but rather felt as a focus of resistance to medial advancement. The amount of tissue that must be divided in this way depends very much on the breadth of the $\mathrm{V}$ and the amount of flap advancement required to approximate its two limbs. A broad V necessitates more extensive soft -tissue division; a narrow V, less. Division must be continued until the flap can be advanced to close the defect with a minimum of tension. The extent of mobilization required also depends to some extent on the preexisting laxity of the eyelids and local tissues generally. When the amount of soft -tissue division is considerable, the flap may ultimately appear to be attached inferiorly by little more than the skin and immediately subcutaneous tissues superficially and the conjunctiva deeply. There should be no particular concern about this . In contrast to the cheek rotation flap used in lower eyelid reconstruction, with its recognized vulnerability to necrosis, the ratio of length to breadth and the relative smallness of the transposed flap make it safe.

\section{Management of the Conjunctiva}

When the lateral canthotomy has been carried out, the conjunctiva provides no resistance to medial advancement and does not need to be formally divided. There is an abundance of lax conjunctiva in the lateral fornix that allows unimpeded lateral movement of the eyeball. As the flap is drawn medially, the conjunctiva spreads out to line the skin and muscle that are being advanced to form the reconstructed segment of the eyelid. Their free margins lie side by side and, when sutured together, will form the margin of the reconstructed lid.

\section{Method of Suture}

The method used to close the $\mathrm{V}$ defect is designed to avoid exposing suture material within the conjunctival sac. With the margins of the $\mathrm{V}$ approximated, suturing is carried out in two layers, the tarsal plate and the conjunctiva behaving as a single structure. When the tarsal plates have been accurately approximated, the conjunctiva heals quickly and without incident. The plates can be effectively sutured together with interrupted 6-0 chromic catgut on an atraumatic needle, placing the knots on the muscle side of the tarsal plate.

As part of this approximation, it is essential to match the lid margins carefully, and care in this respect is continued in the skin closure, using identification points such as the gray line and the eyelashes. The reconstruction is completed by suturing together the free margins of the flap and the conjunctiva. The sutures used to approximate the tarsal plates have a very low tensile strength, and it is important to make sure that there is no tension on the suture line.

\section{Completion of the Z-Plasty}

At the time of initial elevation of the transposed flap in preparation for its transfer, the triangular flap designed as part of the Z-plasty is also elevated, in readiness to be rotated downward into the triangular secondary defect that opens up when the transposed flap is advanced medially. When this triangular flap has been rotated into the defect and sutured in position, suturing of the skin is completed lateral to the outer canthus. In most elderly patients there is considerable laxity in the tissue above the suture line, and some adjustment in tension and trimming of the upper margin may be required to make the suture line lie neatly. 

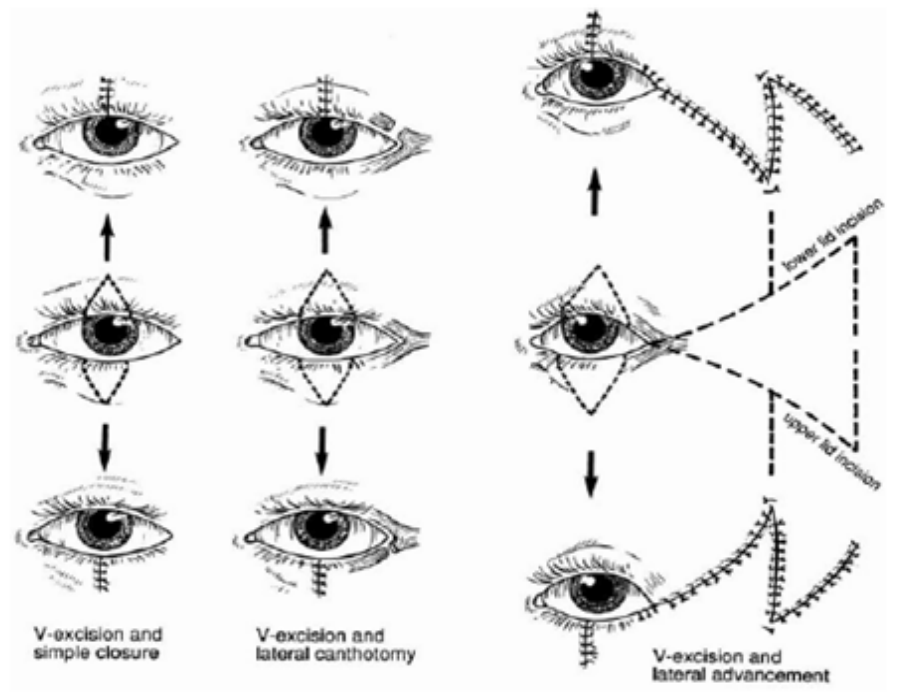

Figure 1: Showing diagrammatic representation of $\mathrm{V}$ shaped excision then and Mc gregor flap.

\section{Case Report}

A 55 year old female patient presented with swelling over the left lower eyelid and lateral periorbital area. On examination after removal of swelling it was revealed that there was complete loss of lateral $2 / 3^{\text {rd }}$ of lower eyelid including the skin, subcutaneous tissue, tarsal plate. There was extensive skin loss in the lateral periorbital area. The upper eyelid was absolutely normal. The whole flap was elevated according to the outline sketched over the skin, and thorough hemostasis was performed. The apex between the donor and the receptor area is sutured first near the medial canthus, followed by the margin with orbicularis oculi fibres, thus completing the anterior lamella. The repair was done in 2 layers. The patient recovered smoothly without any significant post operative complications. In subsequent follow up, the patient did not have any major problems like irritation of the eyes, epiphora, synechia or corneal ulcers.

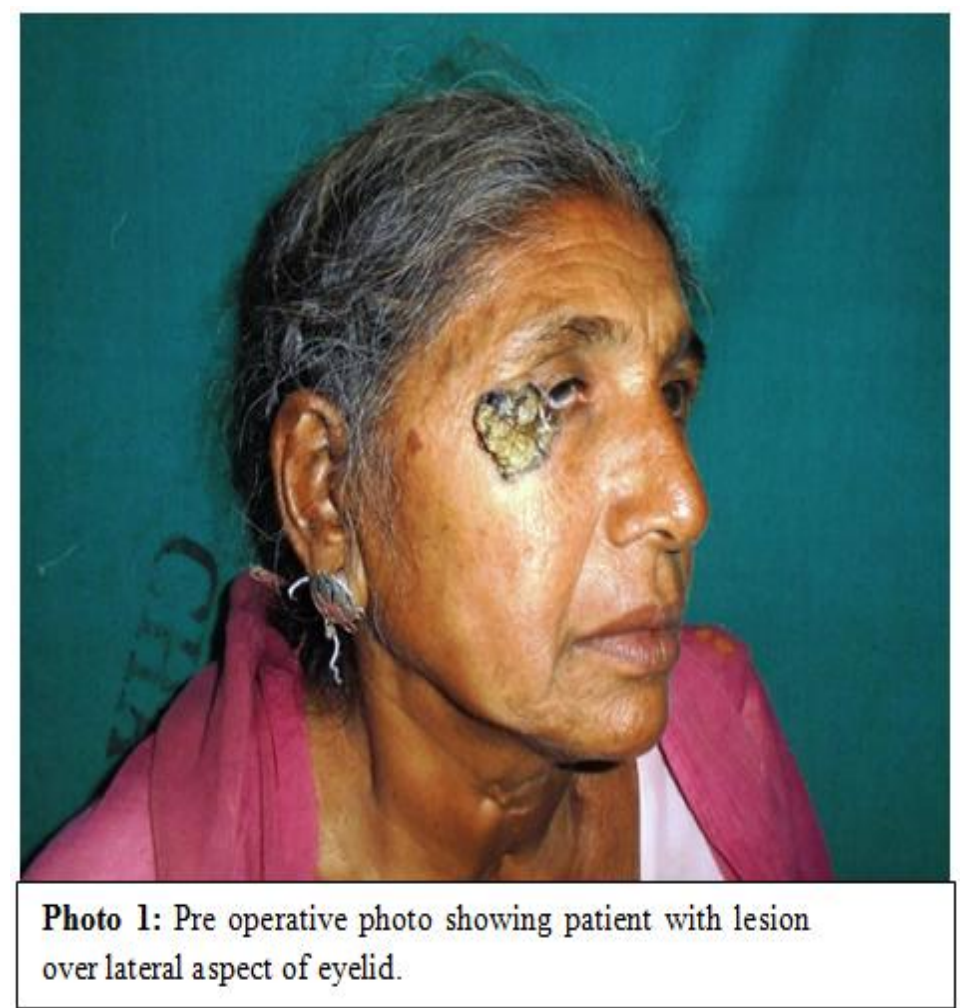




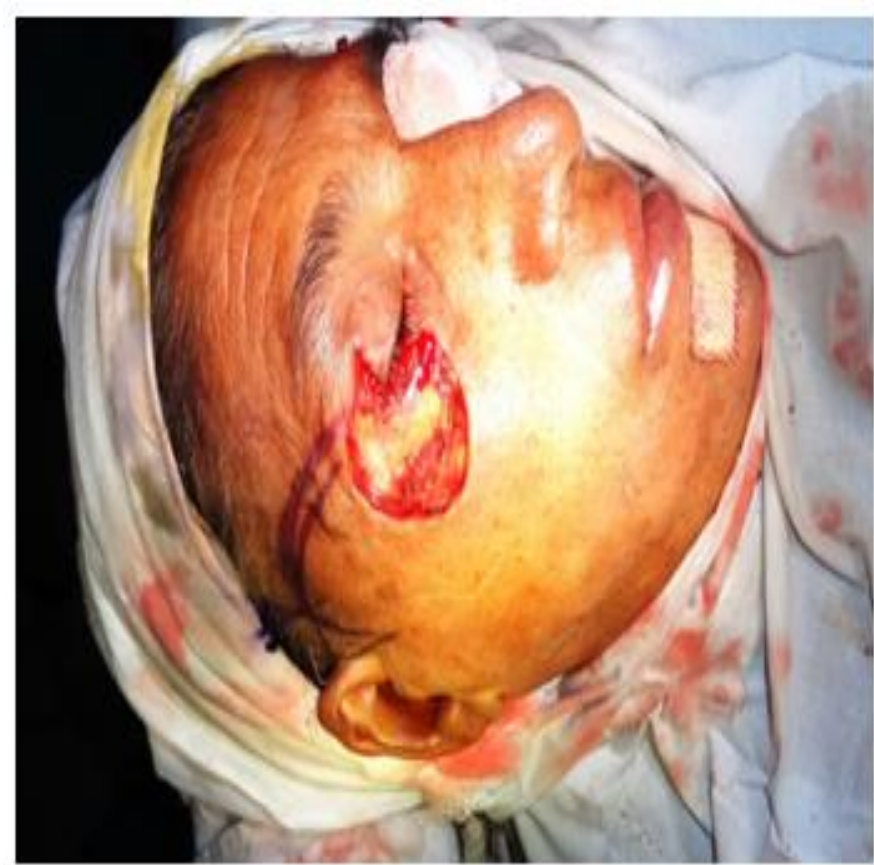

Photo 2: Intra op photo after excision of lesion.

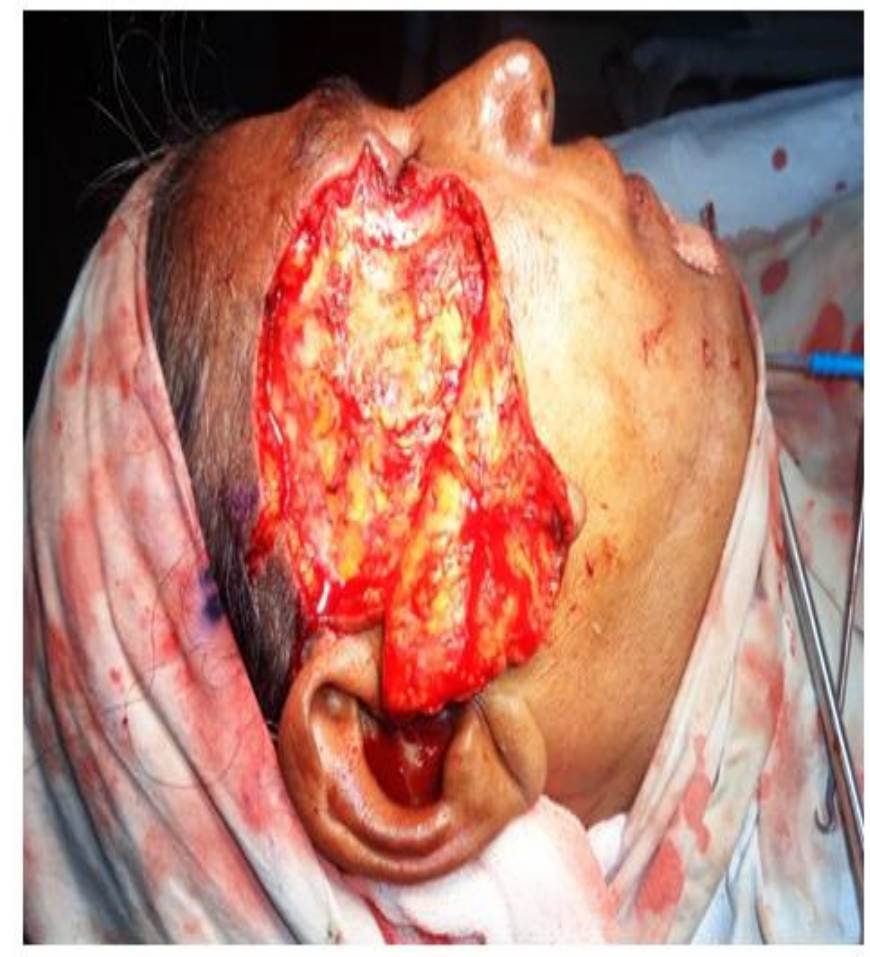

Photo 3: Intra op photo showing elevation of flap as well as $\mathrm{Z}$ plasty incision. 


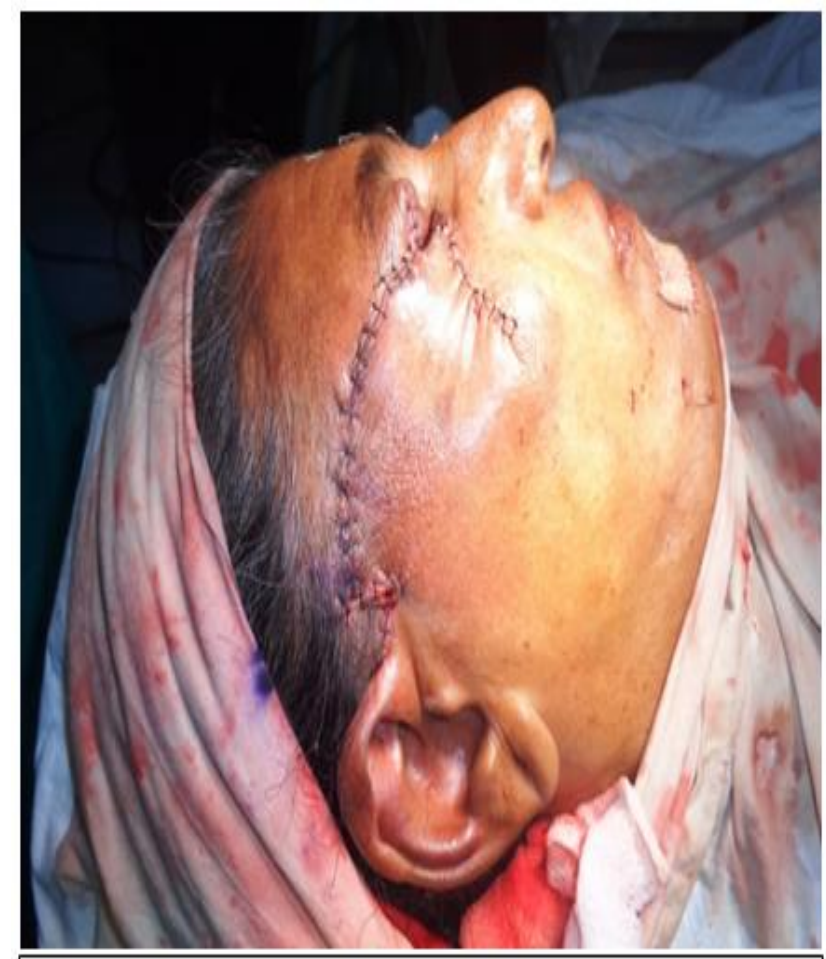

Photo 4: Intra op photo showing suture in situ with good suture line.

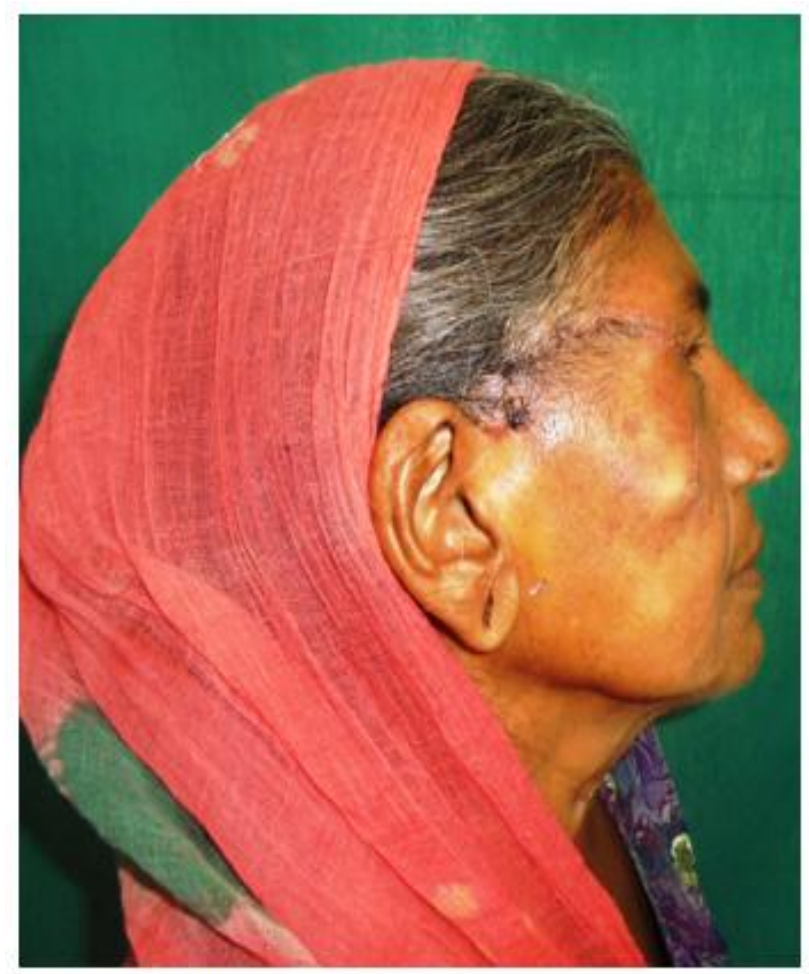

Photo 5: Post op photo showing suture removal with good suture line on lateral view. 


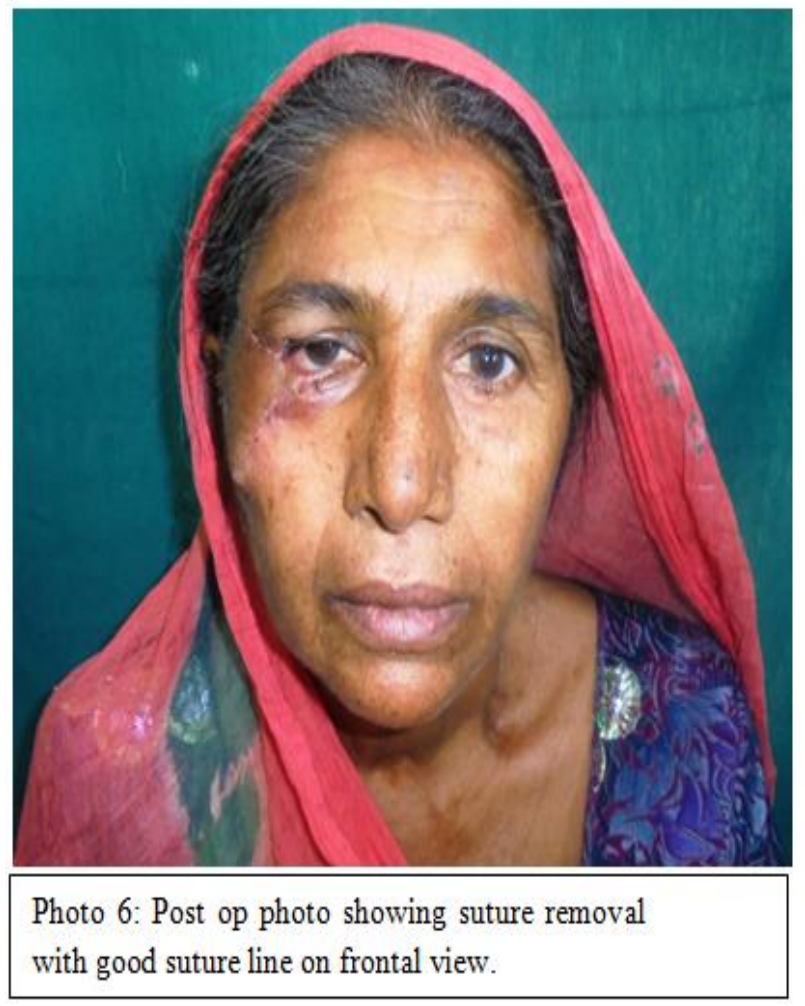

\section{Summary}

Probably the greatest virtues of this technique are its simplicity, safety, and reliability, as well as the fact that it is applicable to either eyelid. Provided the flap is designed correctly and the reconstruction is not pushed beyond its proper limits, particularly as used in the upper eyelid, it gives little trouble.

\section{References}

[1]. McGregor IA. Eyelid reconstruction following subtotal resection of upper or lower eyelid. Br J Plast Surg 1973;26:346.

[2]. McGregor IA, McGregor FM. Cancer of the face and mouth. Edinburgh: Churchill Livingstone, 1986. 